\title{
The Promotion of Virtual Reality Technology in Branded Agricultural Products
}

\author{
Jun-Jun YAN ${ }^{1, a}$ and Xin-Yu GUO ${ }^{2, b, *}$ \\ ${ }^{1}$ No.11, Shuguang Garden Middle Road, Haidian District, Beijing,China \\ ${ }^{2}$ No.11, Shuguang Garden Middle Road, Haidian District, Beijing,China \\ a575528671@qq.com, bguoxy@nercita.org.cn
}

Keywords: Virtual Reality, Agricultural Products, Promotion.

\begin{abstract}
This article main introduces the concept of virtual reality technology, the research status at domestic and foreign. It also analyzes the development status of China's branded agricultural products and expounds the problems existing in the development of branded agricultural products. By combining virtual reality technology with branded agricultural products, and using modern methods to promote branded agricultural products, thereby promoting the development of branded agricultural products.
\end{abstract}

\section{Introduction}

As a computer interface technology, virtual reality technology can provide users with visual, auditory, and other intuitive real-time sensory interaction methods to maximize user convenience, thereby reducing the burden on users and improving the efficiency of the system.

The three-dimensional spatial performance capability, the human-computer interaction operating environment and the immersive feeling brought about by people have changed the boring provided by virtual reality, changed the boring and passive situation between people and computer.

Virtual reality technology has opened up new research fields in the human-computer interaction interface and providing a new description method for the visualization of data of various projects. At the same time, it provides great convenience for people to explore the macro-world and micro-world, and other things that are not convenient for direct observation [1].

\section{Virtual Reality Technology}

\section{The Concept of Virtual Reality Technology}

Virtual reality technology can simulate human behavior in the natural environment, so we can say that virtual reality technology is an advanced human-computer interaction technology. Virtual reality technology can generate a realistic virtual environment through computer technology, including visual, auditory, tactile and other perceptions.

In the virtual space provided, the user can freely move and observe the surrounding scenery at will, so that the user can perceive and study the changing laws of the objective world in the virtual world. For virtual reality technology, what is more important is the participation of users. 
Users can talk to the virtual world and feel the immersion of the virtual world. It can be said that users are the core of virtual reality technology [1].

\section{Research Status}

\section{Foreign Research Status}

As the birthplace of virtual reality technology, the United States initially applied virtual reality technology to the military's simulation training for flight pilots and astronauts. After the Cold War, with the reduction of US military spending, virtual reality technology has gradually turned into civilian technology. At present, NASA has established an aviation VR training system, a space station VR training system, etc., and has created a nationwide VR education system.

In Europe, the UK is ahead of other European countries in terms of virtual reality technology development, especially in the areas of parallel processing, auxiliary equipment design and application research. Bristol, the company in British focuses on the intersection of virtual reality technology applications on the overall integrated technology, and they are leading in some areas of software and hardware [2].

\section{Research State in China}

Compared with developed countries, there is still a certain gap in the research on virtual reality technology in China. But it must be said that virtual reality technology has attracted a certain degree of attention and attention in China, and has been of interest to people from all walks of life.

As one of the earliest units to study virtual reality technology in China, the Virtual Realization and Visualization New Technology Laboratory of the Department of Computer Science of Beijing University of Aeronautics and Astronautics integrates a distributed virtual environment. They also created the virtual reality demonstration environment, the virtual reality system for pilot training and the development platform for virtual reality application systems, and the like.

In addition, the Department of Computer Science of Beijing University of Aeronautics and Astronautics also focuses on the representation and processing of physical properties of objects in virtual environments, developing some hardware in the visual interface of virtual reality and propose related algorithms and implementation methods [2].

\section{Current Status of Agricultural Product Development in China}

\section{The Brand of Agricultural Products is Messy and the Scale is Small}

For China, most of the agricultural products are not registered with the trademark; the proportion of trademark registrations that result in agricultural products is small in the total number of trademark registrations. And a huge number of the agricultural products have the phenomenon of brand mess and small scale.

\section{Less Well-Known Brands, Weak Brand Influence}

Due to the development of branding of agricultural products, the number of branded agricultural products is increasing rapidly. 
Although many primary agricultural products have registered trademarks, most of them only focus on the identification function of trademarks, which do not reflect the deep value of brands, and lack the uniqueness and exclusivity of branded agricultural products.

\section{Lack of Scientific and Technological Content and Added Value}

Most of Chinese agricultural products are primary processed products, and the scientific and technological content and added value are not high, which leads to the lack of secondary value-added products in Chinese agricultural products.

\section{Lack of Effective Mechanisms to Operate the Brand}

On the whole, the choice of branded agricultural products lacks uniform standards. Because of the lack of brand selection, follow-up management is not in place, neglecting the planning and promotion of branded products, which making it difficult to establish the image of brand. It is necessary to establish and improve the corresponding brand agricultural product mechanism so that brand recognition can effectively respond to market and consumer choices [3].

\section{The Role of Virtual Reality Technology in the Promotion of Agricultural Products}

Promoting the development of branding of agricultural products is the fundamental solution for the prosperity of the industry and the construction of a modern agricultural industrial system. A brand is a business name and a symbol, which is used to identify a product or service of a producer or operator, and differentiate other similar products or services.

A brand is an important intangible asset, a reputation and a promise. The brand of agricultural products represents the quality commitment made by the producers or operators of agricultural products to consumers. Because of its unique cultural connotation, it has become the main way of competition for agricultural products.

Promoting the development of branding of agricultural products is an inevitable requirement for deepening the structural reform of the supply side of agriculture and transforming the mode of agricultural development.

It is an important means to improve the core competitiveness of agricultural products and the comprehensive competitiveness of agriculture, and a fundamental measure to achieve agricultural development, rural prosperity and farmers' income.

\section{Establish Agricultural Database}

With the advent of the era of big data, the collection and analysis of data has affected the development of agricultural products to some extent. Through virtual reality technology, an agricultural database can be created, and users can learn about agricultural products in the database, including understanding crop information, obtaining growth patterns of crops, and pests and diseases and more. By obtaining information from the database, users can diagnose their crops and obtain corresponding governance methods.

\section{Cultivate New Varieties and Improve Varieties}

In order to improve the quality of agricultural products and the yield of corresponding crops, it is necessary to continuously cultivate high-yield new varieties of crops.

The traditional method of cultivation is through continuous practice of improvement, planting the varieties, and obtaining results after a long period of growth and development. 
However, by establishing a virtual breeding laboratory, it is possible to simulate different plant types of crops through virtual reality technology. Then, in the virtual environment that set by people, the final optimal plant type is selected after continuous experimentation. The use of virtual reality technology solves the external factors that may lead to errors in breeding, thus achieving the goal of scientific breeding [4].

\section{Develop Virtual Farms, Entertain and Educate}

The virtual farm is a three-dimensional game developed based on virtual reality technology, which mainly includes the cultivation and receipt of crops, and the processing and transportation of agricultural products. Users can plant virtual crops in virtual land. In the process of planting harvested crops, users can learn basic knowledge about crop planting and agricultural product processing. Virtual farms allow users to learn in the game and have fun in learning, thus creating a deep interest in agriculture.

\section{Establish an Agricultural Digital Museum to Inherit Traditional Agricultural Culture}

China has a long history and culture in agriculture. The development of agriculture is not only the development of agricultural technology, but also the inheritance of traditional agricultural culture. With the acceleration of China's urbanization process; many people do not fully understand agriculture. Establish a Agricultural Digital Museum, combining multimedia and virtual reality technology, vividly displaying relevant agricultural products and agricultural knowledge, can effectively publicize agricultural scientific knowledge and spread traditional Chinese agricultural culture.

\section{Conclusion}

Virtual reality technology in China is in the stage of development and improvement. As Chinese requirements for agricultural products and agricultural modernization are getting higher and higher, the requirements for virtual reality technology will also increase.Virtual reality technology is widely used in agriculture and agricultural products. The rapid development of network technology will definitely drive the development of virtual reality technology. By applying virtual reality technology to the development of agricultural products, virtual reality technology will provide a new breakthrough for the development of agricultural products with its unique advantages.

\section{References}

[1] Xingen Hu. Overview of Virtual Reality Technology [J]. Science and Technology Plaza, 2007, (5): 236-238.

[2] Wei Xu. Research status and development of virtual reality technology at home and abroad [J]. Modern Business and Industry, 2009, (2): 279-280

[3] Mingyue Zhang. Discussion on the Development of Agricultural Products Brand in China [J]. Journal of Rural Economy and Technology, 2017, 28(9): 130-131

[4] Pei Zhang, Lili Zhang, Dandan Cheng. Exploring the role of virtual reality technology in agricultural extension [J]. Industry and Technology Forum, 2015, 14(18): 45-46 
[5] Pei Yang. Virtual Reality Technology and Its Application in Agriculture [J]. Anhui Agricultural Science Bulletin, 2008(14): 23-24.

[6] Meimei Zhu, Chunxiao Zhu, Sijia Lin. Research on Portable Remote Immersion Visualization Technology and Device [J]. Modern Agriculture, 2014(2): 6-7 